\title{
Identification of candidate genes, regions and markers for pre-harvest sprouting resistance in wheat (Triticum aestivum L.)
}

Adrian L Cabral ${ }^{1,3}$, Mark C Jordan ${ }^{1 *}$, Curt A McCartney ${ }^{1}$, Frank M You ${ }^{1}$, D Gavin Humphreys ${ }^{1}$, Ron MacLachlan ${ }^{2}$ and Curtis J Pozniak ${ }^{2}$

\begin{abstract}
Background: Pre-harvest sprouting (PHS) of wheat grain leads to a reduction in grain yield and quality. The availability of markers for marker-assisted selection (MAS) of PHS resistance will serve to enhance breeding selection and advancement of lines for cultivar development. The aim of this study was to identify candidate regions and develop molecular markers for PHS resistance in wheat. This was achieved via high density mapping of single nucleotide polymorphism (SNP) markers from an Illumina $90 \mathrm{~K}$ Infinium Custom Beadchip in a doubled haploid (DH) population derived from a RL4452/'AC Domain' cross and subsequent detection of quantitative trait loci (QTL) for PHS related traits (falling number [FN], germination index [G] and sprouting index [SI]). SNP marker sequences flanking QTL were used to locate colinear regions in Brachypodium and rice, and identify genic markers associated with PHS resistance that can be utilized for MAS in wheat.

Results: A linkage map spanning $2569.4 \mathrm{cM}$ was constructed with a total of 12,201 SNP, simple sequence repeat (SSR), diversity arrays technology (DArT) and expressed sequence tag (EST) markers. QTL analyses using Multiple Interval Mapping (MIM) identified four QTL for PHS resistance traits on chromosomes 3B, 4A, 7B and 7D. Sequences of SNPs flanking these QTL were subject to a BLASTN search on the International Wheat Genome Sequencing Consortium (IWGSC) database (http://wheat-urgi.versailles.inra.fr/Seq-Repository). Best survey sequence hits were subject to a BLASTN search on Gramene (www.gramene.org) against both Brachypodium and rice databases, and candidate genes and regions for PHS resistance were identified. A total of 18 SNP flanking sequences on chromosomes 3B, 4A, 7B and 7D were converted to KASP markers and validated with matching genotype calls of Infinium SNP data.
\end{abstract}

Conclusions: Our study identified candidate genes involved in abscissic acid (ABA) and gibberellin (GA) metabolism, and flowering time in four genomic regions of Brachypodium and rice respectively, in addition to 18 KASP markers for PHS resistance in wheat. These markers can be deployed in future genetic studies of PHS resistance and might also be useful in the evaluation of PHS in germplasm and breeding material.

Keywords: Wheat, Pre-harvest sprouting, Quantitative trait loci, Candidate genes

\footnotetext{
* Correspondence: mark.jordan@agr.gc.ca

${ }^{1}$ Cereal Research Centre, Agriculture and Agri-Food Canada, Morden, MB

R6M 1Y5, Canada

Full list of author information is available at the end of the article
}

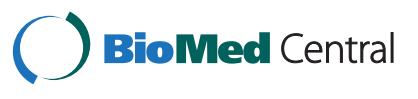

(c) 2014 Cabral et al.; licensee BioMed Central Ltd. This is an Open Access article distributed under the terms of the Creative Commons Attribution License (http://creativecommons.org/licenses/by/4.0), which permits unrestricted use, distribution, and reproduction in any medium, provided the original work is properly credited. The Creative Commons Public Domain Dedication waiver (http://creativecommons.org/publicdomain/zero/1.0/) applies to the data made available in this article, unless otherwise stated. 


\section{Background}

Preharvest sprouting is observed across all major wheat growing regions in the world. In western Canada, the average annual losses due to PHS are approximately $\$ 100$ million [1]. Insufficient seed dormancy is one major factor contributing to pre-harvest sprouting losses, particularly under humid, wet weather conditions at harvest. PHS resistant/tolerant wheat cultivars and land races have been identified globally, with origins mainly in Canada, USA, Australia, China, Japan, South Africa, Kenya and New Zealand [2]. Canadian red-seeded spring wheat cultivars (AC Domain, AC Majestic, Columbus, Pasqua, Waskada, Harvest) and white spring wheat genotypes (AC Vista, Snowbird, Snowstar, Kanata, HY361) are known to carry resistance to PHS, all having derived their resistance alleles from a red-seeded breeding line RL4137 $[1,3]$.

Of the three PHS traits, $F N[4,5]$ is most commonly used to quantify PHS [6] and indirectly measures the activity of the enzyme $\alpha$-amylase that breaks down starch in germinating grains. Degradation of grain-starch as the result of greater $\alpha$-amylase activitys result in lower $F N$ values and are an indirect indication of low levels of PHS resistance or dormancy. Two other important traits for the characterization of PHS are GI $[7,8]$ and SI [9]. While GI values deduced from seed-germination tests in petri dishes are a direct measure of seed dormancy, $S I$ values obtained via artificial wetting of intact wheat spikes, detect dormancy and properties of the inflorescence that affect PHS [5].

Quantitative trait loci (QTL) linked to PHS traits have been reported on all 21 hexaploid wheat chromosomes [10-13], mainly on 3A [14-17], 3B and 3D [17-19], 4A [2,20-24], 5A [25,26], 6B and 7D [27]. Of these, the PHS QTL on $4 \mathrm{~A}$ has consistently been identified in several different mapping populations. The RL4452/'AC Domain' $\mathrm{DH}$ population has been extensively characterized for QTL detection of PHS [28], agronomic [29] and quality traits [30], in several past studies that involved a small number of molecular markers. These studies relied mainly on SSR marker data for the preparation of genetic maps and locating QTL on chromosomes. With low costs and rapid advancements in sequencing technology, thousands of molecular markers, mainly SNPs have become available in wheat. Additionally, access to genome sequence information for rice [31] and Brachypodium [32] will now facilitate comparative mapping for the identification of genes underlying various important quantitative traits in wheat.

Interaction among PHS QTL (QxQ, QTL epistasis), and the environment $(\mathrm{QxE}, \mathrm{QxQxE})$ have been reported from various studies [18,33-35] aimed at understanding the complex genetic structure of QTL. As chromosomal locations of PHS QTL are not uniform across populations, obtaining a consensus on the precise genomic location of important trait QTL is required for fine mapping and cloning studies. Meta-QTL or Meta-analysis [36] integrates several QTL studies of a common trait to provide a meaningful estimate of the exact location and number of QTL for that given trait. Eight PHS QTL on chromosomes 3A, 3B, 3D and 4A were identified in a Meta-QTL study [37] involving 15 different populations (five DH; nine recombinant inbred line [RIL]; one backcross [BC]).

A high level of genome-synteny exists among wheat, Brachypodium and rice, with wheat being more closely related to Brachypodium than to rice [38,39]. Conservation or collinearity of genetic markers $[40,41]$ and greater structural similarities in the coding regions of orthologous genes [39] of wheat and Brachypodium have been reported. However, given differences in gene content in orthologous regions of wheat, Brachypodium [41] and rice [42], it might be beneficial to use both genomic sequences of Brachypodium and rice in comparative mapping studies for map based cloning and gene discovery in wheat.

Our study deployed SNP markers from a 90K Infinium iSelect Custom Beadchip [43], in addition to available SSR, DArT and ESTs, to generate high density genetic maps for the identification of PHS resistance QTL. Sequences corresponding to polymorphic SNPs flanking PHS QTL were analyzed against genomic sequences of Brachypodium and rice. The objectives of our research were a) to identify candidate genes and regions in Brachypodium and rice that are orthologous to PHS resistance QTL intervals in wheat, and b) to utilize sequences of SNPs flanking PHS QTL to develop KASP markers for MAS of PHS resistance.

\section{Results}

\section{Linkage mapping}

A total of 12,201 SNP, SSR, DArT and EST markers were mapped to all 21 wheat chromosomes. The resulting linkage map spanning $2569.4 \mathrm{cM}$ is reported in Additional file 1. Of the 12,201 markers, 11,282 or 92.5\% were SNPs, while the remaining 919 or $7.5 \%$ comprised SSR, DArT and EST markers. The largest number of SNP markers $(6,291)$ were distributed across the B genome, followed by 4,125 SNPs mapped to the A genome, and 1,785 SNP markers on the $\mathrm{D}$ genome (Table 1).

\section{QTL analysis}

PHS datasets were analyzed with both MIM and simple interval mapping (SIM; data not shown) methods. As results of both methods were very similar, only those of MIM were reported in this study. The MIM [44] analysis identified four QTL with significant effects, located across chromosomes 3B, 4A, 7B and 7D. Each of these four QTL appeared in two or more environments and had peak LOD scores greater than the critical threshold LOD at $5 \%$ significance levels $\left(\alpha_{0.05}\right)$ [45]. Coincident 
Table 1 Cumulative map-lengths of A, B and D genome chromosomes alongside corresponding genome-wise distribution of SNP markers mapped in the hexaploid DH population of RL4452/'AC Domain'

\begin{tabular}{lccccc}
\hline Genome & Map length (cM) & Mapped markers & SNPs & SSRs, DArTs \& ESTs & \% SNPs \\
\hline A & 888.4 & 4125 & 3816 & 309 & 92.5 \\
B & 940.6 & 6291 & 5871 & 420 & 93.3 \\
D & 740.4 & 1785 & 1595 & 190 & 89.4 \\
(A+B + D) & 2569.4 & 12201 & 11282 & 919 & 92.5 \\
\hline
\end{tabular}

QTL for GI, SI and FN were located on chromosome 4A. Across trials, RL4452 alleles on $3 \mathrm{~B}$ and $7 \mathrm{~B}$ provided PHS resistance as they reduced SI. However, 'AC Domain' alleles also provided PHS resistance as they increased $F N$ on 4A and 7D (with the exception of the Glenlea 2005 trial in which they reduced $F N$ on 7D) and reduced $S I$ and GI on 4A (Table 2).

\section{Candidate regions and genes for PHS resistance}

Sequences of SNPs flanking QTL for PHS resistance on chromosomes 3B, 4A, 7B and 7D were subjected to BLASTN searches on the IWGSC and Gramene databases and returned hits to candidate regions in Brachypodium and rice (Table 3). Genetic and physical maps displaying orthologous regions for PHS resistance in wheat, Brachypodium and rice are given in Figures $1 \mathrm{a}$ and b. A $7.8 \mathrm{cM}$ QTL interval on chromosome $3 \mathrm{~B}$ was orthologous to a $\sim 7.0 \mathrm{Mb}$ region $(46,936,013$ - 53,904,697 bp) on chromosome 2 of Brachypodium (Bradi2) and to a $\sim 8.7 \mathrm{Mb}$ $(27,906,608$ - 36,656,340 bp) region on chromosome 1 of rice (Os01). The 4A QTL interval was $12.2 \mathrm{cM}$ and was orthologous to a $\sim 0.52 \mathrm{Mb}$ region $(481,247-1,030,837 \mathrm{bp})$ on chromosome 1 of Brachypodium (Bradi1) and to a $~ 6.9 \mathrm{Mb}(29,401,950$ - 36,320,679 bp) region on chromosome 3 of rice (OsO3). On chromosome 7B.1, the QTL interval spanned $1.7 \mathrm{cM}$ and was orthologous to a $\sim 1.8 \mathrm{Mb}$ region $(42,620,688-44,420,413 \mathrm{bp})$ on chromosome 1 of Brachypodium (Bradi1) and to a $\sim \mathrm{Mb}$ $(5,588,196-6,603,975 \mathrm{bp})$ region on chromosome 6 of rice (Os06). The QTL interval on 7D.2 was $7.7 \mathrm{cM}$ and was orthologous to a $\sim 2.0 \mathrm{Mb}$ region $(47,249,027$ $49,335,697 \mathrm{bp})$ on chromosome 1 of Brachypodium (Bradi1), and a $\sim 0.5 \mathrm{Mb}$ region $(2,558,015$ - 3,079,059 bp) on chromosome 6 of rice (Os06).

Table 2 Results of Multiple Interval Mapping (MIM): four QTL for PHS traits (GI, SI, FN) identified on chromosomes 3B, 4A, 7B.1 and 7D.2 in a DH population of RL4452/'AC Domain' replicated in multi-year environments (Glenlea and Winnipeg in Manitoba; Swift Current in Saskatchewan)

\begin{tabular}{|c|c|c|c|c|c|c|c|}
\hline QTL & Trial dataset & Chromosome (Linkage gp.) & QTL peak location (cM) & Additive $^{a}$ & $\% \mathrm{PV}\left(R^{2}\right)$ & LOD & $a 0.05$ \\
\hline \multicolumn{8}{|c|}{ Germination Index (GI) } \\
\hline QGi.crc-4A & Glenlea2005 & $4 \mathrm{~A}$ & 59.3 & -0.04 & 27.6 & 12.83 & 3.86 \\
\hline QGi.crc-4A & Winnipeg2004 & $4 \mathrm{~A}$ & 59.5 & -0.05 & 58.1 & 34.56 & 3.93 \\
\hline QGi.crc-4A & Winnipeg2005 & $4 \mathrm{~A}$ & 59.4 & -0.02 & 29.6 & 13.93 & 3.86 \\
\hline \multicolumn{8}{|c|}{ Sprouting Index (SI) } \\
\hline QSi.crc-3B & Glenlea2005 & $3 B$ & 63.6 & 0.43 & 12.7 & 5.39 & 3.96 \\
\hline QSi.crc-3B & Winnipeg2004 & $3 B$ & 70.2 & 0.53 & 16.1 & 6.97 & 3.95 \\
\hline QSi.crc-4A & Glenlea2005 & $4 \mathrm{~A}$ & 59.3 & -0.57 & 20.5 & 9.12 & 3.96 \\
\hline QSi.crc-4A & Winnipeg2004 & $4 \mathrm{~A}$ & 56.8 & -0.85 & 32.1 & 15.38 & 3.95 \\
\hline QSi.crc-4A & Winnipeg2005 & $4 \mathrm{~A}$ & 58.0 & -0.44 & 12.7 & 5.41 & 3.90 \\
\hline QSi.crc-4A & Swift Current2003 & $4 \mathrm{~A}$ & 58.0 & -0.49 & 10.5 & 4.41 & 3.94 \\
\hline QSi.crc-7B & Swift Current2003 & 7B.1 & 55.6 & 0.78 & 20.5 & 9.12 & 3.94 \\
\hline QSi.crc-7B & Swift Current2004 & 7B.1 & 56.4 & 0.59 & 11.8 & 4.99 & 3.92 \\
\hline \multicolumn{8}{|c|}{ Falling Number (FN) } \\
\hline QFn.crc-4A & Glenlea2005 & $4 \mathrm{~A}$ & 64.2 & 22.49 & 11.2 & 4.71 & 3.83 \\
\hline QFn.crc-4A & Winnipeg2004 & $4 \mathrm{~A}$ & 56.2 & 45.45 & 25.8 & 11.85 & 3.95 \\
\hline QFn.crc-7D & Glenlea2003 & 7D.2 & 18.9 & 33.40 & 13.2 & 5.64 & 3.99 \\
\hline QFn.crc-7D & Glenlea2005 & 7D.2 & 20.2 & -33.49 & 20.6 & 9.19 & 4.13 \\
\hline
\end{tabular}

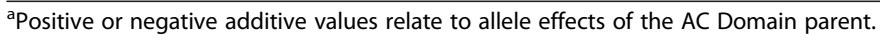


Table 3 Genetic map locations of SNP markers flanking PHS QTL on chromosomes 3B, 4A, 7B.1 and 7D.2 in a wheat DH population of a RL4452/'AC Domain' cross and their corresponding physical locations/candidate regions in Brachypodium distachyon and rice

\begin{tabular}{|c|c|c|c|c|}
\hline SNP marker & $\begin{array}{l}\text { Map } \\
\text { (cM) }\end{array}$ & $\begin{array}{l}\text { Survey sequence } \\
\text { Contig no. }\end{array}$ & $\begin{array}{l}\text { BLASTN hits to Brachypodium genes } \\
\text { (genomic regions in parenthesis) }\end{array}$ & $\begin{array}{l}\text { BLASTN hits to Rice genes } \\
\text { (genomic regions in parenthesis) }\end{array}$ \\
\hline & & Chromosome 3B & & \\
\hline wsnp_Ku_c6825_11858665 & 63.0 & 10469056 & Bradi2g46510 $(46,936,013-46,952,333)$ & LOC_Os01g48680 $(27,906,608-27,920,980)$ \\
\hline wsnp_Ex_c4769_8510104 & 63.0 & 10613849 & Bradi2g46590 $(47,003,547-47,009,237)$ & LOC_Os01g48790 $(27,983,688-27,990,383)$ \\
\hline RAC875_rep_c113906_294 & 64.0 & 10557485 & Bradi2g51030 (50,699,047-50,702,962) & LOC_Os01g56200 $(32,367,683-32,371,816)$ \\
\hline BobWhite_c46650_260 & 64.0 & 10441023 & Bradi2g51017 (50,685,769-50,695,622) & LOC_Os01g56190 $(32,350,513-32,360,765)$ \\
\hline Kukri_c4310_489 & 64.6 & 10759762 & Bradi2g51040 (50,703,620-50,708,573) & LOC_OsO2g13910 $(7,558,777-7,568,835)$ \\
\hline \multirow[t]{2}{*}{ TA002966-0294 } & 65.1 & 10635317 & Bradi2g46710 $(47,135,003-47,136,451)$ & LOC_Os01g56580 $(32,615,694-32,622,894)$ \\
\hline & & 10712014 & Bradi2g49590 $(49,632,703-49,638,684)$ & LOC_Os01g54100 $(31,111,291-31,116,151)$ \\
\hline BS00078127_51 & 65.7 & 10754454 & Bradi2g51530 $(51,119,031-51,123,083)$ & LOC_Os01g56810 $(32,788,487-32,792,751)$ \\
\hline Kukri_c21818_519 & 66.2 & 10455881 & Bradi2g51620 $(51,191,828-51,198,372)$ & LOC_Os01g56910 (32,869,293-32,878,216) \\
\hline wsnp_Ra_rep_c74606_72470419 & 66.8 & 10523702 & Bradi2g51710 (51,287,497-51,313,181 & LOC_Os01g57082 $(32,984,982-32,994,519)$ \\
\hline IACX3871 & 66.8 & 10521243 & Bradi2g51890 $(51,441,692-51,446,044)$ & LOC_Os01g57450 $(33,200,667-33,201,485)$ \\
\hline Excalibur_c73633_120 & 67.3 & 10673653 & Bradi2g48430 $(48,731,037-48,732,308)$ & LOC_Os01g52260 $(30,042,527-30,043,938)$ \\
\hline wsnp_Ex_c5547_9774195 & 68.4 & 10770075 & Bradi2g53020 $(52,250,581-52,257,598)$ & LOC_Os01g59670 $(34,514,117-34,520,887)$ \\
\hline wsnp_Ex_rep_c69664_68618163 & 68.4 & 10477393 & Bradi2g52540 $(51,883,735-51,889,623)$ & LOC_Os01g58680 $(33,919,393-33,924,664)$ \\
\hline wsnp_Ku_rep_c72700_72370664 & 69.0 & 10484009 & Bradi2g53340 $(52,475,967-52,481,992)$ & LOC_Os01g60180 $(34,803,492-34,804,046)$ \\
\hline RAC875_rep_c116515_181 & 69.0 & 1068363 & Bradi2g53130 $(52,329,608-52,334,764)$ & LOC_Os01g59880 $(34,629,359-34,635,205)$ \\
\hline BobWhite_rep_c64944_264 & 69.6 & 1040995 & Bradi2g53970 $(52,969,054-52,973,550)$ & LOC_Os01g61400 $(35,505,448-35,508,543)$ \\
\hline Tdurum_contig38427_237 & 70.2 & 10658322 & Bradi2g55100 $(53,817,575-53,821,406)$ & LOC_Os01g63250 $(36,656,340-36,660,768)$ \\
\hline Tdurum_contig27495_111 & 70.2 & 10538814 & Bradi2g53450 $(52,567,117-52,569,109)$ & LOC_Os01g60430 $(34,946,618-34,949,027)$ \\
\hline \multirow[t]{2}{*}{ Kasp3B(survey)_17 } & 70.8 & 10495803 & Bradi2g55230 $(53,904,697-53,906,640)$ & LOC_Os03g60200 $(34,238,474-34,241,647)$ \\
\hline & & Chromosome 4A & & \\
\hline BS00068243_51 & 53.8 & 7023446 & Bradi2g12660 $(11,006,410-11,009,518)$ & LOC_Os01g28244 $(15,823,709-15,829,849)$ \\
\hline CD920298 & 58.6 & 7174272 & Bradi1g00600 $(481,247-482,062)$ & LOC_Os03g64290 $(36,320,679-36,333,253)$ \\
\hline \multirow[t]{2}{*}{ Kukri_c12563_52 } & 59.3 & 7128338 & Bradi1g51817 (50,293,482-50,308,189) & LOC_Os05g37500 $(21,943,044-21,959,786)$ \\
\hline & & & Bradi1g00760 (565,638-570,467)! & LOC_Os03g63370 $(35,809,964-35,814,672) !$ \\
\hline BS00072025_51 & 59.3 & 7168762 & Bradi1g00730 (555,714-559,377) & LOC_Os03g64210 $(36,281,400-36,283,271)$ \\
\hline RAC875_C21369_425 & 59.8 & 7070429 & Bradi1g00820 $(594,037-597,877)$ & LOC_Os03g64190 $(36,265,672-36,271,489)$ \\
\hline IAAV3132 & 59.8 & 7114346 & Bradi1g01007 (695,876-702,209) & LOC_Os03g63920 $(36,110,059-36,119,639)$ \\
\hline wsnp_Ex_c5470_9657856 & 60.4 & 7174581 & Bradi1g01070 (731,493-733,959) & LOC_Os03g51390 $(29,401,950-29,403,115)$ \\
\hline RAC875_c25124_182 & 61.6 & 7061368 & Bradi1g01227 $(825,624-828,017)$ & LOC_Os03g63680 $(35,968,492-35,970,517)$ \\
\hline \multirow[t]{3}{*}{ wsnp_Ku_c4924_8816643 } & 62.7 & 501046 & Bradi1g52230 $(50,605,616-50,611,584)$ & LOC_Os02g29140 $(17,257,940-17,266,066)$ \\
\hline & & 3540051 & Bradi1G00720 (552,185-555,346)! & - \\
\hline & & 864232 & - & LOC_Os03g60710 (34,502,945-34,508,158)! \\
\hline \multirow[t]{3}{*}{ Excalibur_c24511_1196 } & 63.2 & 7119833 & Bradi1g49910 $(48,564,700-48,565,690)$ & LOC_Os06g16640 $(9,564,124-9,566,967)$ \\
\hline & & 7139864 & Bradi1g00820 (594,037-597,877)! & - \\
\hline & & 5949088 & - & LOC_Os03g53500 $(30,679,685-30,689,230) !$ \\
\hline Tdurum_contig13489_292 & 63.8 & 7124315 & Bradi1g01500 $(976,919-979,161)$ & LOC_Os03g63470 $(35,855,445-35,860,549)$ \\
\hline wsnp_JD_c38619_27992279 & 66.0 & 7098863 & Bradi1g01580 (1,030,837-1,034,525) & LOC_Os03g63410 $(35,826,263-35,830,205)$ \\
\hline
\end{tabular}


Table 3 Genetic map locations of SNP markers flanking PHS QTL on chromosomes 3B, 4A, 7B.1 and 7D.2 in a wheat DH population of a RL4452/'AC Domain' cross and their corresponding physical locations/candidate regions in Brachypodium distachyon and rice (Continued)

\begin{tabular}{|c|c|c|c|c|}
\hline & & Chromosome 7B.1 & & \\
\hline CAP7_c10566_170 & 55.3 & 3116911 & Bradi1G46150 (44,420,413-44,423,001)! & LOC_Os06g10710 (5,588,196-5,594,757) \\
\hline BobWhite_rep_c64768_264 & 55.3 & 3032904 & Bradi1G46137 $(44,416,953-44,419,121)$ & LOC_Os06g10760 (5,619,105-5,621,750) \\
\hline Tdurum_contig84962_256 & 55.3 & 3032904 & Bradi1G46137 $(44,416,953-44,419,121)$ & LOC_Os06g10760 (5,619,105-5,621,750) \\
\hline BS00022498_51 & 55.3 & 3115694 & Bradi1G46060 $(44,341,065-44,348,362)$ & LOC_Os06g10880 (5,677,080-5,682,126) \\
\hline wsnp_Ex_c908_1754208 & 56.4 & 3153345 & Bradilg45210 $(43,434,039-43,436,397)$ & LOC_Os06g12270 (6,603,975-6,604,635) \\
\hline \multirow[t]{2}{*}{ Tdurum_contig68347_605 } & 56.4 & 3153345 & Bradi1G45210 $(43,434,039-43,436,397)$ & LOC_Os06g12270 (6,603,975-6,604,635) \\
\hline & & & & LOC_Os06g12280 $(6,605,479-6,608,454)$ \\
\hline RFL_Contig124_558 & 57 & 3126436 & Bradi1g44967 $(43,073,188-43,080,744) !$ & - \\
\hline \multirow[t]{2}{*}{ BobWhite_c46772_564 } & 57 & 3109791 & Bradi1G44860 $(42,951,596-42,953,323)$ & LOC_Os06g12990 (7,118,829-7,120,448) \\
\hline & & & Bradi1G44850 $(42,949,245-42,951,551)$ & - \\
\hline GENE-4333_211 & 57 & 3153554 & Bradi1G44790 $(42,899,346-42,900,477)$ & - \\
\hline \multirow[t]{2}{*}{ Tdurum_contig51087_573 } & 57 & 3165147 & Bradi1G44440 $(42,620,688-42,629,717)$ & LOC_Os06g13820 (7,661,691-7,670,035) \\
\hline & & Chromosome 7D.2 & & \\
\hline RAC875_c1829_321 & 14.3 & 3849095 & Bradilg48660 $(47,326,685-47,327,292)$ & LOC_Os06g06460 $(3,040,092-3,041,121)$ \\
\hline Kukri_c32845_116 & 14.3 & 3964075 & Bradilg50860 (49,335,697-49,339,907) & LOC_Os06g05660 (2,558,015-2,562,242) \\
\hline TA002473-0717 & 14.3 & 3929478 & Bradi1g49140 $(47,871,489-47,874,424)$ & LOC_Os06g05700 $(2,579,088-2,581,726)$ \\
\hline wsnp_CAP8_rep_c9647_4198594 & 22.0 & 3945994 & Bradi1g48610 $(47,249,027-47,255,499) !$ & LOC_Os06g06560 $(3,079,059-3,086,808)$ \\
\hline
\end{tabular}

'Weak hit to genomic regions in Brachpodium or rice that is orthologous to the QTL interval for PHS resistance in wheat.

Best hits that do not correspond to the candidate region in Brachpodium or rice are in italics.

Brachypodium/rice candidates for PHS resistance orthologous to consensus regions on wheat chromosomes 3B, $4 \mathrm{~A}, 7 \mathrm{~B}$ and 7D (Additional file 2) were identified. In the 3B region there are 895 genes in the Brachypodium orthologous region and 1375 in the rice region. The $4 \mathrm{~A}$ region had 98 genes in the Brachypodium region and 1159 in rice, while the 7B region had 148 in Brachypodium and 155 in rice and the 7D region had 235 in Brachypodium and 88 in rice. Genes involved in ABA and GA metabolism as well as those affecting flowering time were present in the QTL regions. Among these were Bradi2g49795/ Os01g54490 (FT PEBP [phosphatidylethanolamine - binding protein] homologous to Flowering Locus T gene), Os01g61100, Os01g63030 (Far-red impaired responsive [FAR1] family protein) orthologous to chromosome 3B, Bradi1g00950/Os03g63970 (gibberellin 20 [GA20] oxidase putative expressed protein), Os03g56630, Os03g62660 (Far-red impaired responsive [FAR1] family protein) orthologous to 4A, Bradilg46060/Os06g10880 (ABF3/ ABF2 - abscissic acid responsive elements) orthologous to 7B, Bradilg48640, Bradilg48650, Bradi1g48822, Bradi1g48816 (Far-red impaired responsive [FAR1] family protein), Bradilg48690, Bradi1g50240 (VRN1-AP2/B3 - like transcriptional factor family protein) and Bradilg48830/ Os06g06320 (Vrn3/FT PEBP [phosphatidylethanolamine binding protein] homologous to Flowering Locus T gene) orthologous to chromosome 7D.

\section{Development and validation of KASP primers}

A total of 18 KASP markers, five each for chromosomes $3 \mathrm{~B}$ and 7B.1, and four each for chromosomes 4A and 7D.2 (Table 4) were developed from sequences of SNPs flanking QTL for PHS resistance. Genetic map locations of individual KASP markers were identical to the respective SNP from which they were derived. Primer sets of all 18 KASP markers are listed in Additional file 3. Further, we validated the conversion of these 18 KASPs from matching genotype calls of Infinium SNP data on $183 \mathrm{DH}$ progeny genotypes. Four DH progeny genotypes of the RL4452/'AC Domain' cross were identified to carry PHS resistance on chromosomes 3B, 4A, 7B and 7D (Additional file 4).

\section{Discussion}

The objectives of our research were to identify candidate regions for PHS resistance QTL of wheat and develop KASP markers (for MAS) from sequences of SNPs flanking such QTL. This is an important step in the process of map-based cloning of genes that underlie important quantitative traits like PHS resistance. Our objectives were achieved using 11,282 SNPs from the $90 \mathrm{k}$ Infinium Custom Beadchip to develop a high density linkage map in the RL4452/'AC Domain' mapping population and subsequently detect QTL for PHS resistance on chromosomes 3B, 4A, 7B and 7D. Comparative mapping utilizing 
a) PHS resistance: Orthologous regions in Wheat, Brachypodium and Rice

a)_WHEAT.Chm3B_(Distance:cM)

Mbp

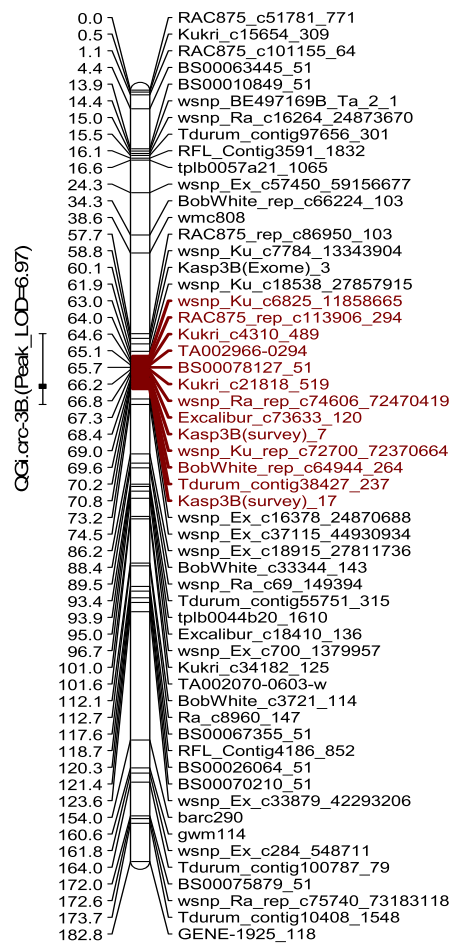

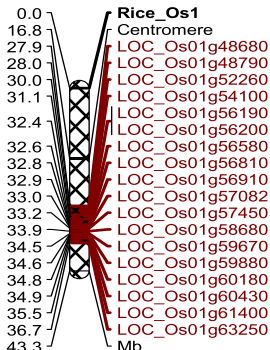

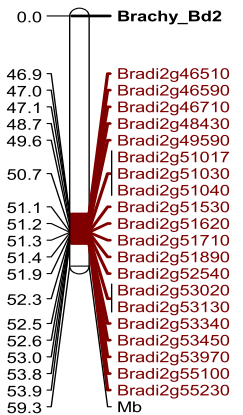

b) WHEAT.Chm7B.1 (Distance:cM)

Mbp

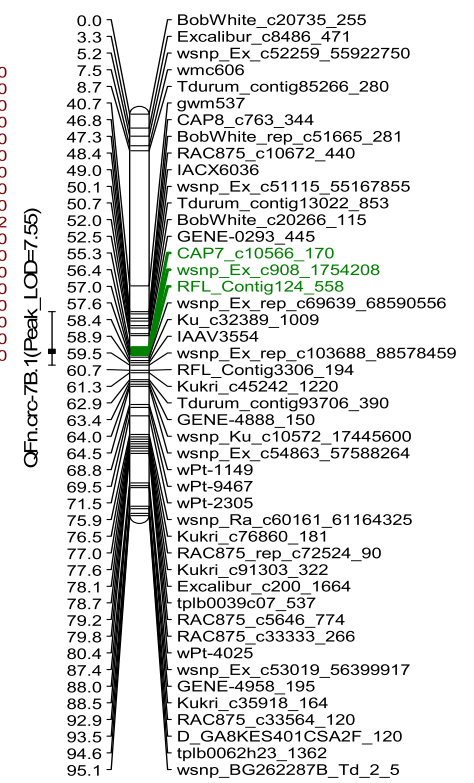

b) PHS resistance: Orthologous regions in Wheat, Brachypodium and Rice

a)_WHEAT.Chm4A_(Distance:cM)

Mbp

b)_WHEAT.Chm7D.2_(Distance:cM) Mbp
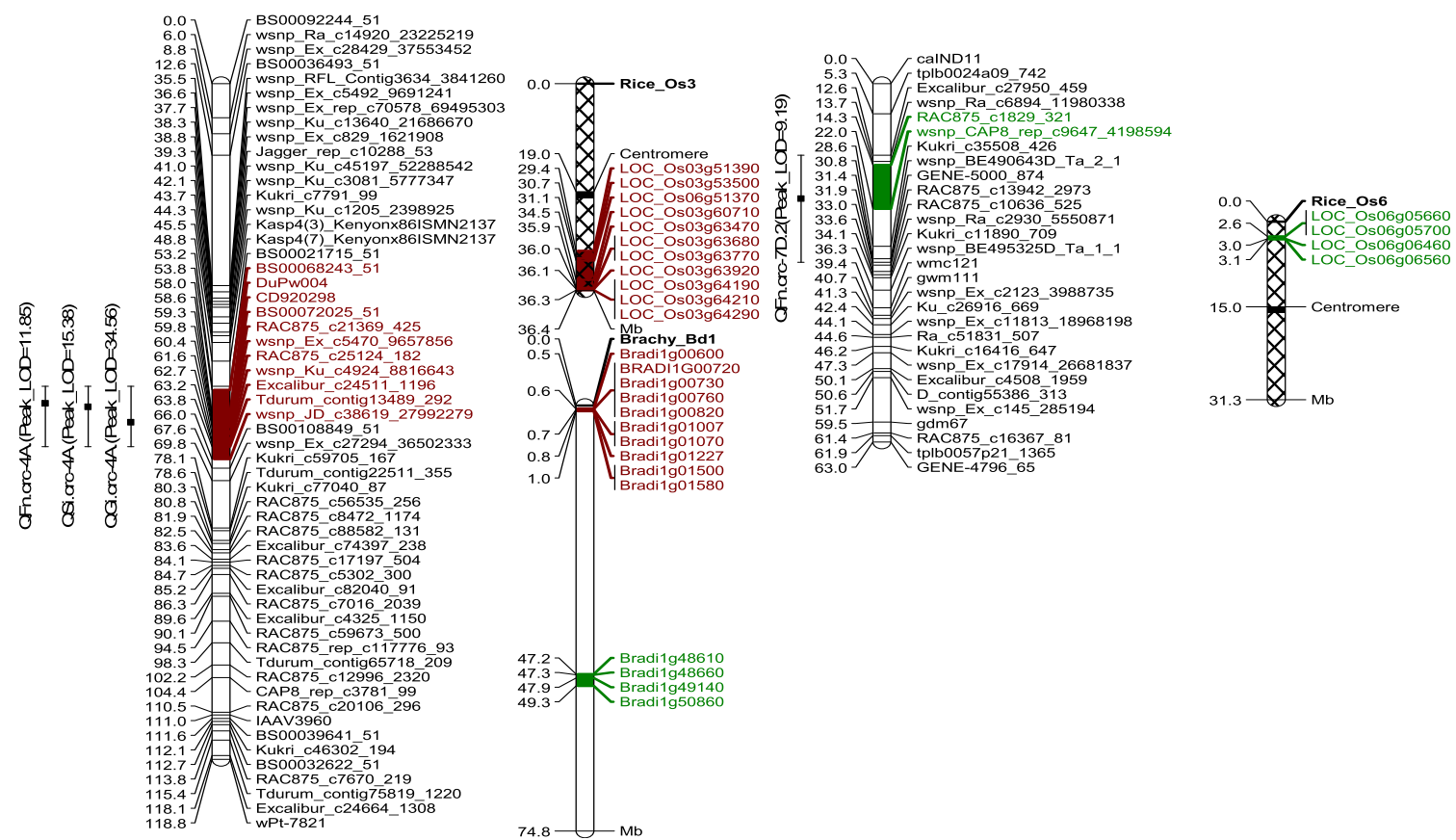

Figure 1 (See legend on next page.) 
(See figure on previous page.)

Figure 1 Location of QTL and syntenic regions in Brachpodium and rice. a. Location of QTL and flanking markers for PHS resistance on a) wheat chromosome 3B and its candidate regions on Brachypodium Bd2 and rice Os 1, and $\mathbf{b}$ ) chromosome 7B.1 and its candidate regions on Brachypodium Bd1 and rice Os6. b. Location of QTL and flanking markers for PHS resistance on a) wheat chromosome $4 \mathrm{~A}$ and its candidate regions on Brachypodium Bd1 and rice Os3, and $\mathbf{b})$ chromosome 7D.2 and its candidate regions on Brachypodium Bd1 and rice Os6.

sequences of SNPs flanking PHS resistance QTL enabled identification of candidate genes and regions in Brachypodium and rice. The resulting 18 KASP markers can be deployed in future genetic studies of PHS, and in evaluation of PHS in germplasm and breeding material.

Of the 12,201 mapped markers, 11,282 or $92.5 \%$ were SNP markers, while the remaining 919 or $7.5 \%$ were SSR, DArT and EST markers. The B genome chromosomes accounted for the largest number of 6291 SNP markers, followed by the A genome with 4125 SNPs, and the D genome with 1785 SNP markers. A likely explanation for larger numbers of B genome SNP markers could be the greater genetic diversity of $\mathrm{B}$ genome species when compared to the $\mathrm{A}$ and $\mathrm{D}$ genome species $[46,47]$. A faster rate of evolution of the B genome due to greater polymorphism and duplication events, in addition to greater genetic diversity brought about by cross pollination were cited [48-50] as possible explanations for findings of a greater number of ESTs associated with more unique loci on the $B$ genome when compared to the $\mathrm{A}$ and $\mathrm{D}$ genomes.
PHS datasets were analyzed with both MIM and SIM (data not shown) methods. Because results of both methods were very similar, only those of the MIM analyses were reported. As QTL identified using MIM were robust and supported by SIM results, it is unlikely that additional large effect QTL involved in epistatic interactions might have been detected using other QTL mapping methods that detect both main effect (M-QTL) and epistatic QTL (E-QTL). Further, a Meta-QTL study [37] reporting PHS QTL on 4A and group 3 chromosomes support significant PHS QTL identified on chromosome 3B and 4A of our study.

The most consistent of the four PHS QTL identified on chromosomes 3B, 4A, 7B and 7D were located on chromosome 4A; GI, SI and FN trait QTL each accounting for $58.1 \%, 32.1 \%$ and $25.8 \%$ of the phenotypic variation in their respective traits. The QTL for these PHS traits were coincident and maybe associated with the same gene(s). These findings might suggest that chromosome 4A is involved in regulation of PHS trait QTL in our test population. Previous reports of the association of PHS

Table 4 A list of 18 Competitive Allele-Specific PCR (KASP) markers developed for MAS of PHS from SNPs flanking PHS QTL on chromosomes 3B, 4A, 7B and 7D in a DH population of a RL4452/'AC Domain' cross

\begin{tabular}{|c|c|c|c|c|}
\hline SI. & KASP marker & Source SNP & Chr & PHS trait \\
\hline 1. & Kasp3B_wsnp_Ku_rep_c72700_72370664 & wsnp_Ku_rep_c72700_72370664 & $3 B$ & $S /$ \\
\hline 2. & Kasp3B_RAC875_rep_c116515_181, & RAC875_rep_c116515_181, & $3 B$ & SI \\
\hline 3. & Kasp3B_BobWhite_rep_c64944_264 & BobWhite_rep_c64944_264 & $3 B$ & SI \\
\hline 4. & Kasp3B_wsnp_Ex_c16378_24870688 & wsnp_Ex_c16378_24870688 & $3 B$ & SI \\
\hline 5. & Kasp3B_RAC875_c530_354 & RAC875_c530_354 & $3 B$ & SI \\
\hline 6. & Kasp4A_BS00072025_51 & BS00072025_51 & $4 \mathrm{~A}$ & $G I, S I, F N$ \\
\hline 7. & Kasp4A_Kukri_c12563_52 & Kukri_c12563_52 & $4 \mathrm{~A}$ & $G I, S I, F N$ \\
\hline 8. & Kasp4A_RAC875_c21369_425 & RAC875_C21369_425 & $4 \mathrm{~A}$ & $G I, S I, F N$ \\
\hline 9. & Kasp4A_wsnp_Ex_c16175_24619793 & wsnp_Ex_c16175_24619793 & $4 \mathrm{~A}$ & $G I, S I, F N$ \\
\hline 10 & Kasp7B_wsnp_Ex_c908_1754208 & wsnp_Ex_c908_1754208 & 7B.1 & SI \\
\hline 11 & Kasp7B_RFL_Contig124_558 & RFL_Contig124_558 & 7B.1 & SI \\
\hline 12 & Kasp7B_RAC875_c1638_165 & RAC875_C1638_165 & 7B.1 & $S /$ \\
\hline 13 & Kasp7B_wsnp_Ex_rep_c69639_68590556 & wsnp_Ex_rep_c69639_68590556 & 7B.1 & $S /$ \\
\hline 14 & Kasp7B_Ku_c32389_1009 & Ku_c32389_1009 & 7B.1 & SI \\
\hline 15 & Kasp7D_Excalibur_c22419_460 & Excalibur_c22419_460 & 7D.2 & $F N$ \\
\hline 16 & Kasp7D_RAC875_c1829_321 & RAC875_C1829_321 & 7D.2 & FN \\
\hline 17 & Kasp7D_Kukri_c32845_116 & Kukri_c32845_116 & 7D.2 & FN \\
\hline 18 & Kasp7D_wsnp_CAP8_rep_c9647_4198594 & wsnp_CAP8_rep_c9647_4198594 & 7D.2 & FN \\
\hline
\end{tabular}


traits with chromosome 4A [2,20-24], support the importance of this QTL for PHS

In addition to a major SI QTL on 4A, two other QTL for SI were identified on chromosomes $3 \mathrm{~B}$ and 7B.1. Both SI QTL on 3B and 7B.1 were detected in two of six environments. QTL that provide tolerance to late maturity $\alpha$ - amylase (LMA) have been mapped on 3BS and $7 \mathrm{BL}$ in an Australian wheat cross Cranbrook/Halberd [51]. In both studies, the SSR markers Xwmc623, Xwmc808, Xgwm72, Xwmc612, Xgwm285, Xwmc693, $X w m c 1$ (3B LMA QTL interval) and Xgwm577, Xwmc273, Xwmc276 (7B LMA QTL interval) also flanked corresponding PHS QTL intervals on chromosomes 3B.1 and 7B.1 respectively (data not shown). Further, alleles of a regulator gene $V p-1 B$ on $3 \mathrm{~B}$ have been reported to influence grain dormancy in Chinese wheat varieties [19]. In a follow up study [52], the $V P-1 B$ locus was validated in a white-grained Chinese landrace Wanxianbaimaizi (high seed dormancy and PHS tolerance) using SSR markers and a gene-specific primer $V p 1$. A CIM analysis identified a seed dormancy QTL QSd.ahau-3B on 3B flanked by $V p 1$ which is linked to an SSR marker Xwmc446 that also happens to flank the PHS QTL interval on chromosome $3 \mathrm{~B}$ of our study. The above findings suggest that PHS and LMA QTL on chromosomes $3 \mathrm{~B}$ and $7 \mathrm{~B}$ are likely the same.

'AC Domain' alleles contributed to increasing the $F N$ on 7D (linkage group 7D.2), with the exception of the Glenlea 2005 trial, wherein a negative additive score was observed for the $F N$. While the FN QTL on chromosome 7D is unique to our study, a significant time to maturity (Mat) QTL (PV $=26 \%$ ) also on 7D, and a positive contribution of the RL4452 allele, has been reported previously by [29] in the same RL4452/'AC Domain' population. The authors reported an SSR marker Xgwm130 tightly linked to this QTL, which is distally located on 7DL, and is $1.1 \mathrm{cM}$ from the QTL peak of our study. In the Glenlea 2005 trial (with a negative additive score for $F N$ ), the average $F N$ (LS Mean) score of $183 \mathrm{DH}$ progeny was the lowest of the four trials (data not shown). The low $F N$ score at this location might suggest greater levels of PHS of 'AC Domain' genotypes, probably brought on by wet weather conditions at the maturity stages or during the three weeks preceding harvest [53]. As QTL locations of both these Mat and FN traits nearly coincide and are influenced by negative and positive additive effects (with the exception of the FN QTL of the Glenlea 2005 trial) of 'AC Domain' alleles respectively, the action of a pleiotrophic locus regulating both $F N$ and Mat could be assumed. At Glenlea in 2005 it is possible that the lower $F N$ for the Domain allele is due to adverse weather conditions at maturity or that the 7D QTL identified here might not actually be a PHS QTL, but rather a pleiotrophic effect of the Mat QTL on PHS.
Flanking marker intervals of a given PHS trait (GI, SI or $F N$ ) QTL were not always the same across trials/datasets. It is quite likely that the respective underlying genes influencing each of these traits are the same; difference in QTL interval location being mainly due to environment or experimental error from differences in class means of individual trial data sets [54]. Alternatively, the possibility of two closely linked loci controlling the same trait cannot be ruled out.

BLASTN searches with sequences of SNP markers flanking PHS QTL on chromosomes 3B, 4A, 7B and 7D revealed candidate regions in Brachypodium and rice genomes. The QTL interval on chromosome 3B was orthologous to regions on Bradi2 and the long arm of Os01, while QTL intervals on chromosomes 4A were orthologous to regions on Bradi1 and the short arm of Os03. QTL intervals on chromosome 7B.1 and 7D.2 were orthologous to regions on Bradi1 and the short arm of $\mathrm{OsO6}$ of rice. The above findings of orthology between wheat/rice chromosomes: $3 \mathrm{~B} / \mathrm{Os} 01,4 \mathrm{~A} / \mathrm{Os} 03$ and 7B\&7D/Os06 concur with previous reports [42,55-57] of wheat/rice chromosomal region similarities revealed via comparative mapping with DNA probes and ESTs. Further, orthologies between PHS QTL intervals of 4A, 7B, $7 \mathrm{D}$ and genomic regions of Bradi1, and 3B/Bradi2 in our study will be refined to tease out individual genes responsible for variation in PHS resistance. The availability of information on whole-genome 454 assembled gene sequences of Chinese spring [58] and gene-orthologies among the said wheat and Brachypodium chromosomes established using 5003 ESTs mapped to wheat deletion bins [32] will serve as useful references to complement our efforts.

Eighteen KASP markers were developed from SNP sequences flanking QTL for PHS resistance. Identical genotype calls of Infinium SNP data enabled validation of the 18 KASP markers and identified four (of 183) progeny genotypes of the RL4452/'AC Domain' population possessing PHS resistance on all four QTL on 3B, 4A, 7B and 7D (Additional file 4). Criteria for selection of these genotypes was based on findings of our study: 'AC Domain' (allele 'A') reduced GI and SI on 4A, increased $F N$ on $4 \mathrm{~A}$ and 7D, while RL4452 (allele 'B') reduced SI on chromosomes $3 \mathrm{~B}$ and $7 \mathrm{~B}$. Further, these $18 \mathrm{KASP}$ markers can be deployed in future genetic studies of PHS, and in evaluation of PHS in germplasm and breeding material.

Genes present in Brachypodium and rice in orthologous regions corresponding to the QTL were identified (Additional file 2). The $3 \mathrm{~B}$ region is large and contains over 800 genes in Brachypodium and over 1300 in rice. More markers are needed to reduce the size of the region and the emerging reference sequence of chromosome 3B (http://wheat-urgi.versailles.inra.fr/ 
Seq-Repository/Reference-sequence) will be a valuable resource. There are a number of ABA-inducible genes ( 2 Brachypodium and 3 rice) which could be a starting point to search for additional markers.

The $4 \mathrm{~A}$ and $7 \mathrm{~B}$ regions contain many fewer genes in Brachypodium and rice than the 3B region. Gibberellin 20 oxidase (GA20 - oxidase) [59] on Bradi1/Os03 orthologous to $4 \mathrm{~A}$ and abscissic acid responsive elements (ABF2, ABF3) [60-62] on Bradi1/Os06 orthologous to chromosome $7 \mathrm{~B}$ are candidates worth further study. GA20 - oxidase has previously been considered as a candidate gene underlying PHS QTL on 4A [63].

On chromosome 7D the QTL was coincident with a previously identified maturity QTL in the same population (29). Genes affecting flowering time are present in the orthologous regions in Brachypodium and rice. These include the Far-red impaired responsive (FAR1) related proteins [64] on chromosome Bradi1, as well as VRN1AP2/B3-like transcription factors [65,66] on Bradi1 and phosphatidylethanolamine - binding protein (PEBP) homologous to the Flowering Locus $\mathrm{T}$ gene $[67,68]$ on Bradi1/Os06, orthologous to chromosome 7D.

Because our study utilized a large number of sequencebased SNPs not available for previous mapping studies, the resulting genetic maps and QTL flanking SNP markers are a novel and current resource for identification of underlying genes based on synteny and collinearity to model species Brachypodium and rice. Further, the identification of candidate genes and regions for PHS in Brachypodium and rice will enable a targeted focus for selection of candidate genes whose physiological/biological functions are linked to or influence variation in PHS traits under study. Such candidate gene-specific PCR markers will be developed and validated via mapping to the QTL intervals for PHS resistance in wheat.

\section{Conclusions}

In our study we utilized SNPs from a wheat $90 \mathrm{~K}$ Infinium iSelect Custom Beadchip that permitted detection and assignment of significant PHS resistance QTL to specific chromosomal locations on genetic maps. Sequences of SNPs flanking PHS resistance QTL enabled identification of candidate genes and regions for PHS in Brachypodium and rice via comparative mapping. The 18 KASP markers resulting from this study can be suitably deployed in future genetic studies of PHS and might also be useful in the evaluation of PHS in germplasm and breeding material.

\section{Methods}

Plant material, experimental layout and trait phenotyping A total of $193 \mathrm{DH}$ progeny genotypes derived from a cross RL4452/'AC Domain' were used to develop the genetic linkage map. Of these, trait data was available on
$183 \mathrm{DH}$ lines for detection of QTL across the genome. Data on three PHS traits (GI, SI and FN) was collected from six trials: Glenlea (2003; 2005), Winnipeg (2004; 2005) and Swift Current (2003; 2004), in Manitoba and Saskatchewan Canada. The phenotyping methods, experimental design and layout for each of these traits are described in [6,28].

\section{Molecular markers and genotyping Infinium SNPs and PCR based markers}

The $90 \mathrm{~K}$ Infinium iSelect Custom Wheat Beadchip identified 12,351 polymorphic markers that were added to existing SSR, DArT and EST markers for the RL4452/ 'AC Domain' cross. Of these, a total of 12,201 markers (11282 SNPs; 919 SSRs, DArTs and ESTs) were used in the construction of genetic maps. Further, co-segregating markers were removed from the set of 12,201 markers and QTL analysis was carried out (one marker per bin) with 1054 markers.

\section{Linkage mapping}

Genotypic data of 193 DH progeny, screened with 12,201 markers (SSR, SNP, DArT and ESTs), were used to construct genetic maps for all 21 chromosomes. Bins of co-segregating markers were identified with MSTMap [69], and the most informative marker per bin was retained for mapping with MapDisto ${ }^{\circ}$ [70]. Linkage groups were created using a minimum LOD score of 4 and maximum recombination fraction $(R F)$ of 0.25 . Recombination fractions were converted into centiMorgan (cM) map distances using the Kosambi mapping function.

\section{QTL analysis}

Multi-year trial data collected at six environments on three PHS traits (GI, SI, FN) were used for QTL mapping with QGene version 3.0 software [71]. Trait data and molecular phenotypes of $183 \mathrm{DH}$ progeny assessed with 1054 markers were subject to MIM and SIM (data not shown) analyses. QTL with LOD scores exceeding critical threshold values at $5 \%\left(\alpha_{0.05}\right)$, at two or more environments were deemed significant. Threshold values for trait QTL were obtained through permutation analyses involving 1000 iterations. Further, marker-trait regression $\left(r^{2}\right)$ values were interpreted as the percent phenotypic variation $(\% P V)$ explained due to respective QTL.

\section{Identification of candidate genes and regions in Brachypodium and rice}

Sequences of SNPs flanking QTL for PHS resistance traits (GI, SI, FN) on chromosomes 3B, 4A, 7B and 7D were subject to a BLASTN (Basic search) on the IWGSC database (http://wheat-urgi.versailles.inra.fr/Seq-Repository). Further, best survey sequence hits were subject to a BLASTN search (Maximum E-value 10) on Gramene 
(www.gramene.org) against both Brachypodium and rice databases to obtain candidate regions for PHS resistance. QTL intervals were deduced from centiMorgan map distances between SNP markers flanking QTL peaks of a given PHS resistance trait (GI, SI or $F N)$. Consensus candidate regions for PHS resistance were arrived at from best hits (of PHS QTL flanking SNP sequences) to genes and genomic regions in Brachypodium and rice. A few of the SNP markers returned hits to non-candidate regions/chromosomes prompting the selection of weaker hits to the consensus candidate regions. MapChart 2.2 [72] was used to construct genetic and physical maps of orthologous regions in wheat, Brachypodium and rice. Candidate genes in Brachypodium and rice corresponding to QTL intervals for PHS resistance on chromosomes $3 \mathrm{~B}, 4 \mathrm{~A}, 7 \mathrm{~B}$ and $7 \mathrm{D}$ of wheat were obtained from the online PlantGDB database (http://www.plantgdb. $\operatorname{org} /)$.

\section{KASP markers}

Sequences of SNP markers flanking QTL for PHS resistance on chromosomes $3 \mathrm{~B}, 4 \mathrm{~A}, 7 \mathrm{~B}$ and $7 \mathrm{D}$ were converted to KASP markers. PrimerPicker Lite for KASP version 0.25 (KBioscience ${ }^{\circ}$ ) was used to generate KASP primer sets from QTL flanking SNP sequences. Protocols for the preparation and running of KASP reactions, and PCR conditions are given in the KASP manual (http://www.kbioscience.co.uk/). A FLUOstar Omega plate reader (BMG $\mathrm{LABTECH}^{\circ}$ Offenburg Germany) with KlusterCaller $^{\text {ma }}$ software was used to visualize KASP marker polymorphisms.

\section{Availability of supporting data}

All the supporting data are available as additional files.

\section{Additional files}

Additional file 1: A linkage map constructed using $193 \mathrm{DH}$ progeny genotypes of a RL4452/'AC Domain' cross evaluated with 12,201 polymorphic markers (11282 SNPs and 919 PCR markers).

Additional file 2: Brachypodium and rice candidates corresponding to QTL intervals for PHS resistance on chromosomes 3B, 4A, 7B and 7D in a DH population of a RL4452/'AC Domain' cross.

Additional file 3: List of 18 Competitive Allele-Specific PCR (KASP) primers derived from sequences of SNPs flanking QTL for PHS resistance on chromosomes $3 \mathrm{~B}, 4 \mathrm{~A}, 7 \mathrm{~B}$ and $7 \mathrm{D}$.

Additional file 4: Validation of 18 Competitive Allele-Specific PCR (KASP) markers designed from Illumina iSelect markers flanking QTL in a RL4452/'AC Domain' population.

\section{Competing interests}

The authors declare that they have no competing interests.

\section{Authors' contributions}

MCJ, CAM, ALC (designed and edited the manuscript); ALC, MCJ (conducted the experiment and drafted the manuscript); GH (provided the RL4452/'AC
Domain' DH population and edited the manuscript); CJP (conducted the $90 \mathrm{~K}$ genotyping, edited the manuscript and is the lead of the CTAG project that funded part of this work); FMY, RM (carried out bioinformatics and data sorting work); CAM (performed MST mapping and SNP clustering). All authors read and approved the final manuscript.

\section{Acknowledgements}

This study was conducted as part of the Canadian Triticum Advancement through Genomics (CTAG) project. The authors thank Genome Canada, Genome Prairie, the Western Grains Research Foundation, the Province of Saskatchewan, and Alberta Innovates for funding this project.

\section{Author details}

'Cereal Research Centre, Agriculture and Agri-Food Canada, Morden, MB R6M 1Y5, Canada. ${ }^{2}$ Crop Development Centre, University of Saskatchewan, Saskatoon, SK S7N 5A8, Canada. ${ }^{3}$ National Research Council of Canada, 110 Gymnasium Place, Saskatoon, SK S7N 0W9, Canada.

Received: 17 July 2014 Accepted: 18 November 2014

Published online: 29 November 2014

\section{References}

1. DePauw RM, Knox RE, Singh AK, Fox SL, Humphreys DG, Hucl P: Developing standardized methods for breeding preharvest sprouting resistant wheat, challenges and successes in Canadian wheat. Euphytica 2012, 188:7-14.

2. Ogbonnaya FC, Imtiaz M, DePauw RM: Haplotype diversity of preharvest sprouting QTLs in wheat. Genome 2007, 50:107-118.

3. DePauw RM, Clarke FR, Fofana B, Knox R, Humphreys G, Cloutier S: RL4137 contributes preharvest sprouting resistance to Canadian wheats. Euphytica 2009, 168(3):347-361.

4. Hagberg S: Note on a simplified rapid method for determining alpha-amylase activity. Cereal Chem 1961, 38:202-203.

5. Paterson AH, Sorrells ME, Obendorf RL: Methods of evaluation fo preharvest sprouting resistance in wheat breeding programs. Can J Plant Sci 1989, 69:681-689.

6. Humphreys DG, Noll J: Methods for characterization of preharvest sprouting resistance in a wheat breeding program. Euphytica 2002, 126(1):61-65.

7. Hagemann MG, Ciha AJ: Evaluation of methods used in testing winter wheat susceptibility to preharvest sprouting. Crop Sci 1983, 24:249-253.

8. Reddy LV, Metzger RJ, Ching TM: Effect of temperature on seed dormancy of wheat. Crop Sci 1985, 25:455-458.

9. McMaster GJ, Derera NF: Methodology and sample preparation when screening for sprouting damage in cereals. Cereal Res Commun 1976, 4:251-254

10. Mohan A, Kulwal $P$, Singh R, Kumar V, Mir RR, Kumar J, Prasad M, Balyan HS, Gupta PK: Genome-wide QTL analysis for pre-harvest sprouting tolerance in bread wheat. Euphytica 2009, 168(3):319-329.

11. Munkvold JD, Tanaka J, Benscher D, Sorrells ME: Mapping quantitative trait loci for preharvest sprouting resistance in white wheat. Theor Appl Genet 2009, 119(7):1223-1235.

12. Kulwal PL, Mir RR, Kumar S, Gupta PK: QTL analysis and molecular breeding for seed dormancy and pre-harvest sprouting tolerance in bread wheat. J Plant Biol 2010, 37:59-74.

13. Jaiswal V, Mir RR, Mohan A, Balyan HS, Gupta PK: Association mapping for pre-harvest sprouting tolerance in common wheat (Triticum aestivum L.). Euphytica 2012, 188(1):89-102.

14. Osa M, Kato K, Mori M, Shindo C, Torada A, Miura H: Mapping QTLs for seed dormancy and the Vp1 homologue on chromosome $3 \mathrm{~A}$ in wheat. Theor App/ Genet 2003, 106(8):1491-1496.

15. Kulwal PL, Kumar N, Gaur A, Khurana P, Khurana JP, Tyagi AK, Balyan HS, Gupta PK: Mapping of a major QTL for pre-harvest sprouting tolerance on chromosome 3A in bread wheat. Theor Appl Genet 2005, 111(6):1052-1059.

16. Mori M, Uchino N, Chono M, Kato K, Miura H: Mapping QTLs for grain dormancy on wheat chromosome $3 \mathrm{~A}$ and the group 4 chromosomes, and their combined effect. Theor Appl Genet 2005, 110(7):1315-1323.

17. Fofana B, Humphreys DG, Rasul G, Cloutier S, Brûlé-Babel A, Woods S, Lukow OM, Somers DJ: Mapping quantitative trait loci controlling 
pre-harvest sprouting resistance in a red $\times$ white seeded spring wheat cross. Euphytica 2009, 165(3):509-521.

18. Kulwal PL, Singh R, Balyan HS, Gupta PK: Genetic basis of pre-harvest sprouting tolerance using single-locus and two-locus QTL analyses in bread wheat. Funct Integr Genomic 2004, 4(2):94-101.

19. Chang C, Zhang HP, Feng JM, Yin B, Si HQ, Ma CX: Identifying alleles of Viviparous-1B associated with pre-harvest sprouting in micro-core collections of Chinese wheat germplasm. Mol Breeding 2010, 25(3):481-490.

20. Flintham J, Adlam R, Bassoi M, Holdsworth M, Gale M: Mapping genes for resistance to sprouting damage in wheat. Euphytica 2002, 126(1):39-45.

21. Mares D, Mrva K, Cheong J, Williams K, Watson B, Storlie E, Sutherland M, Zou Y: A QTL located on chromosome 4A associated with dormancy in white-and red-grained wheats of diverse origin. Theor App/ Genet 2005, 111(7):1357-1364.

22. Torada A, Ikeguchi S, Koike M: Mapping and validation of PCR-based markers associated with a major QTL for seed dormancy in wheat. Euphytica 2005, 143(3):251-255.

23. Ogbonnaya FC, Imtiaz M, Ye G, Hearnden PR, Hernandez E, Eastwood RF, Ginkel MV, Shorter SC, Winchester JM: Genetic and QTL analyses of seed dormancy and preharvest sprouting resistance in the wheat germplasm CN10955. Theor App/ Genet 2008, 116(7):891-902.

24. Chen CX, Cai SB, Bai GH: A major QTL controlling seed dormancy and pre-harvest sprouting resistance on chromosome $4 \mathrm{~A}$ in a Chinese wheat landrace. Mol Breeding 2008, 21(3):351-358.

25. Groos C, Gay G, Perretant MR, Gervais L, Bernard M, Dedryver F, Charmet G Study of the relationship between pre-harvest sprouting and grain color by quantitative trait loci analysis in a white $\times$ red grain bread-wheat cross. Theor App/ Genet 2002, 104(1):39-47.

26. Singh R, Matus-Ca'diz M, Baga M, Hucl P, Chibbar RN: Identification of genomic regions associated with seed dormancy in white-grained wheat. Euphytica 2010, 174:391-408.

27. Roy JK, Prasad M, Varshney RK, Balyan HS, Blake TK, Dhaliwal HS, Edwards KJ, Gupta PK: Identification of a microsatellite on chromosomes $6 \mathrm{~B}$ and $\mathrm{a}$ STS on 7D of bread wheat showing an association with preharvest sprouting tolerance. Theor App/ Genet 1999, 99(1):336-340.

28. Rasul G, Humphreys DG, Brûlé-Babel A, McCartney CA, Knox RE, DePauw RM, Somers DJ: Mapping QTLs for pre-harvest sprouting traits in the spring wheat cross RL4452/'AC Domain'. Euphytica 2009, 168(3):363-378.

29. McCartney CA, Somers DJ, Humphreys DG, Lukow O, Ames N, Noll J, Cloutier S, McCallum BD: Mapping quantitative trait loci controlling agronomic traits in the spring wheat cross RL4452 × 'AC Domain'. Genome 2005, 48(5):870-883.

30. McCartney CA, Somers DJ, Lukow O, Ames N, Noll J, Cloutier S, Humphreys DG, McCallum BD: QTL analysis of quality traits in the spring wheat cross RL4452 × 'AC Domain'. Plant Breed 2006, 125(6):565-575.

31. International Rice Genome Sequencing Project: The mapbased sequence of the rice genome. Nature 2005, 436:793-800.

32. The International Brachypodium Initiative: Genome sequencing and analysis of the model grass Brachypodium distachyon. Nature 2010, 463(7282):763-768

33. Liu S, Bai G, Cai S, Chen C: Dissection of genetic components of preharvest sprouting resistance in white wheat. Mol Breeding 2010, 27(4):511-523.

34. Kulwal P, Kumar N, Kumar A, Gupta RK, Balyan HS, Gupta PK: Gene networks in hexaploid wheat: interacting quantitative trait loci for grain protein content. Funct Integr Genomic 2005, 5(4):254-259.

35. Imtiaz M, Ogbonnaya FC, Oman J, van Ginkel M: Characterization of quantitative trait loci controlling genetic variation for preharvest sprouting in synthetic backcross-derived wheat lines. Genetics 2008, 178(3):1725-1736.

36. Goffinet B, Gerber S: Quantitative trait loci: a meta-analysis. Genetics 2000 155(1):463-473.

37. Tyagi S, Gupta PK: Meta-analysis of QTLs involved in pre-harvest sprouting tolerance and dormancy in bread wheat. Triticeae Genomics Genetics 2012, 3(2):9-24.

38. Faris JD, Zhang Z, Fellers JP, Gill BS: Micro-colinearity between rice, Brachypodium, and Triticum monococcum at the wheat domestication locus Q. Funct Integr Genomic 2008, 8(2):149-164.

39. Huo N, Vogel JP, Lazo GR, You FM, Ma Y, McMahon S, Dvorak J, Anderson OD, Luo M-C, Gu YQ: Structural characterization of Brachypodium genome and its syntenic relationship with rice and wheat. Plant Mol Biol 2009, 70(1-2):47-61.
40. Griffiths S, Sharp R, Foote TN, Bertin I, Wanous M, Reader S, Colas I, Moore $\mathrm{G}$ : Molecular characterization of Ph1 as a major chromosome pairing locus in polyploid wheat. Nature 2006, 439:749-752

41. Bossolini E, Wicker T, Knobel PA, Keller B: Comparison of orthologous loci from small grass genomes Brachypodium and rice: implications for wheat genomics and grass genome annotation. Plant J 2007, 49:704-717.

42. Sorrells ME, La Rota M, Bermudez-Kandianis CE, Greene RA, Kantety R, Munkvold JD, Miftahudin, Mahmoud A, Ma X, Gustafson PJ, Qi LL, Echalier B, Gill BS, Matthews DE, Lazo GR, Chao S, Anderson OD, Edwards H, Linkiewicz AM, Dubcovsky J, Akhunov ED, Dvorak J, Zhang D, Nguyen HT, Peng J, Lapitan NLV, Gonzalez-Hernandez JL, Anderson JA, Hossain K, Kalavacharla $V$, et al: Comparative DNA Sequence Analysis of Wheat and Rice Genomes. Genome Res 2003, 13:1818-1827.

43. Wang S, Wong D, Forrest K, Allen A, Chao S, Huang BE, Maccaferri M, Salvi S, Milner SG, Cattivelli L, Mastrangelo AM, Whan A, Stephen S, Barker G, Wieseke R, Plieske J, International Wheat Genome Sequencing Consortium, Lillemo M, Mather D, Appels R, Dolferus R, Brown-Guedira G, Korol A, Akhunova AR, Feuillet C, Salse J, Morgante M, Pozniak C, Luo M-C, Dvorak J, et al: Characterization of polyploid wheat genomic diversity using a high-density 90000 single nucleotide polymorphism array. Plant Biotech J 2014, doi:10.1111/pbi.12183.

44. Kao $\mathrm{CH}$, Zeng ZB, Teasdale RD: Multiple interval mapping for quantitative trait loci. Genetics 1999, 152(3):1203-1216.

45. Churchill GA, Doerge RW: Empirical threshold values for quantitative trait mapping. Genetics 1994, 138:963-971.

46. Chao S, Dubcovsky J, Dvorak J, Luo MC, Baenziger SP, Matnyazov R, Clark DR, Talbert LE, Anderson JA, Dreisigacker S, Glover K, Chen J, Campbell K, Bruckner PL, Rudd JC, Haley S, Carver BF, Perry S, Sorrells ME, Akhunov ED: Population-and genome-specific patterns of linkage disequilibrium and SNP variation in spring and winter wheat (Triticum aestivum L.). BMC Genomics 2010, 11(1):727.

47. Jordan MC, Somers DJ, Banks TW: Identifying regions of the wheat genome controlling seed development by mapping expression quantitative trait loci. Plant Biotech J 2007, 5(3):442-453.

48. Huang S, Sirikhachornkit A, Su X, Faris J, Gill B, Haselkorn R, Gornicki P: Genes encoding plastid acetyl-CoA carboxylase and 3-phosphoglycerate kinase of the Triticum/Aegilops complex and the evolutionary history of polyploid wheat. Proc Natl Acad Sci U S A 2002, 99:8133-8138.

49. Akhunov ED, Akhunova AR, Linkiewicz AM, Dubcovsky J, Hummel D, Lazo GR, Chao S, Anderson OD, David J, Qi L, Echalier B, Gill BS, Miftahudin, Gustafson JP, La Rota M, Sorrells ME, Zhang D, Nguyen HT, Kalavacharla V, Hossain K, Kianian SF, Peng J, Lapitan NLV, Wennerlind EJ, Nduati V, Anderson JA, Sidhu D, Gill KS, McGuire PE, Qualset CO, et al: Synteny perturbations between wheat homoeologous chromosomes caused by locus duplications and deletions correlate with recombination rates. Proc Natl Acad Sci U S A 2003, 100:10836-10841.

50. Qi LL, Echalier B, Chao S, Lazo GR, Butler GE, Anderson OD, Akhunov ED, Dvorak J, Linkiewicz AM, Ratnasiri A, Dubcovsky J, Bermudez-Kandianis CE, Greene RA, Kantety R, La Rota CM, Munkvold JD, Sorrells SF, Sorrells ME, Dilbirligi M, Sidhu D, Erayman M, Randhawa HS, Sandhu D, Bondareva SN, Gill KS, Mahmoud AA, Ma XF, Miftahudin, Gustafson JP, Conley EJ, et al: A Chromosome Bin Map of 16,000 Expressed Sequence Tag Loci and Distribution of Genes Among the Three Genomes of Polyploid Wheat. Genetics 2004, 168:701-712.

51. McNeil MD, Diepeveen D, Wilson R, Barclay I, McLean R, Chalhoub B, Appels R: Haplotype analyses in wheat for complex traits: tracking the chromosome $3 B$ and $7 B$ regions associated with late maturity alpha amylase (LMA) in breeding programs. Crop Pasture Sci 2009, 60(5):463-471.

52. Chang C, Feng JM, Si HQ, Yin B, Zhang HP, Ma CX: Validating a nove allele of viviparous-1 ( $\mathrm{Vp}-1 \mathrm{Bf})$ associated with high seed dormancy of Chinese wheat landrace, Wanxianbaimaizi. Mol Breeding 2010, 25(3):517-525.

53. Mares DJ: Pre-harvest sprouting in wheat. I. Influence of cultivar, rainfall and temperature during grain ripening. Crop Pasture Sci 1993, 44(6):1259-1272.

54. Szalma SJ, Snook ME, Bushman BS, Houchins KE, McMullen MD: Duplicate Loci as QTL. Crop Sci 2002, 42(5):1679-1687.

55. Kurata N, Moore G, Nagamura Y, Foote T, Yano M, Minobe Y, Gale M: Conservation of genome structure between rice and wheat. Nat Biotechnol 1994, 12(3):276-278. 
56. Van Deynze AE, Nelson JC, Yglesias ES, Harrington SE, Braga DP, McCouch SR, Sorrells ME: Comparative mapping in grasses. Wheat relationships. Mol Gen Genet 1995, 248(6):744-754.

57. Gale MD, Devos KM: Comparative genetics in the grasses. Proc Natl Acad Sci U S A 1998, 95(5):1971-1974.

58. Brenchley R, Spannagl M, Pfeifer M, Barker GLA, D'Amore R, Allen AM, McKenzie N, Kramer M, Kerhornou A, Bolser D, Kay S, Waite D, Trick M, Bancroft I, Gu Y, Huo N, Luo M-C, Sehgal S, Gill B, Kianian S, Anderson O, Kersey P, Dvorak J, McCombie WR, Hall A, Mayer KFX, Edwards KJ, Bevan MW, Hall N: Analysis of the bread wheat genome using whole-genome shotgun sequencing. Nature 2012, 491(7426):705-710.

59. Lange T, Hedden P, Graebe JE: Expression cloning of a gibberellin 20-oxidase, a multifunctional enzyme involved in gibberellin biosynthesis. Proc Natl Acad Sci U S A 1994, 91(18):8552-8556.

60. Choi H, Hong J, Ha J, Kang J, Kim S: ABFs, a family of ABA-responsive element binding factors. J Biol Chem 2000, 275:1723-1730.

61. Kim S, Ma J, Perret P, Li Z, Thomas T: Arabidopsis ABI5 subfamily members have distinct DNA binding and transcriptional activities. Plant Physiol 2002, 130:688-697.

62. Kim S, Kang J-Y, Cho D-I, Park JH, Kim SY: ABF2, an ABRE-binding bZIP factor, is an essential component of glucose signaling and its overexpression affects multiple stress tolerance. Plant J 2004, 40:75-87.

63. Tyagi S, Gupta PK: Meta-analysis of QTLs Involved in Pre-harvest Sprouting Tolerance and Dormancy in Bread Wheat. Triticeae Gen Genet 2012, 3:9-24.

64. Hudson M, Ringli C, Boylan MT, Quail PH: The FAR1 locus encodes a novel nuclear protein specific to phytochrome A signaling. Gene Dev 1999, 13(15):2017-2027.

65. Yan L, Loukoianov A, Tranquilli G, Helquera M, Fahima T, Dubcovsky J: Positional cloning of the wheat vernalization gene VRN1. Proc Natl Acad Sci U S A 2003, 100(10):6263-6268.

66. Alonso JM, Stepanova AN, Leisse TJ, Kim CJ, Chen H, Shinn P, Stevenson DK, Zimmerman J, Barajas P, Cheuk R, Gadrinab C, Heller C, Jeske A, Koesema E, Meyers CC, Parker H, Prednis L, Ansari Y, Choy N, Deen H, Geralt M, Hazari N, Hom E, Karnes M, Mulholland C, Ndubaku R, Schmidt I, Guzman P, Aguilar-Henonin L, Schmid M, et al: Genome-wide insertional mutagenesis of Arabidopsis thaliana. Science 2003, 301(5633):653-657.

67. Kojima S, Takahashi Y, Kobayashi Y, Monna L, Sasaki T, Araki T, Yano M: $\mathrm{Hd} 3 \mathrm{a}$, a rice ortholog of the Arabidopsis FT gene, promotes transition to flowering downstream of $\mathrm{Hd} 1$ under short-day conditions. Plant $\mathrm{Cell}$ Physiol 2002, 43(10):1096-1105.

68. Ciaffi M, Paolacci AR, D'Aloisio E, Tanzarella OA, Porceddu E: Identification and characterization of gene sequences expressed in wheat spikelets at the heading stage. Gene 2005, 346:221-230.

69. Wu Y, Bhat PR, Close TJ, Lonardi S: Efficient and Accurate Construction of Genetic Linkage Maps from the Minimum Spanning Tree of a Graph. PLoS Genet 2008, 4(10):e1000212. doi:10.1371/journal.pgen.1000212.

70. Lorieux M: MapDisto: fast and efficient computation of genetic linkage maps. Mol Breeding 2012, in press (doi:10.1007/s1 1032-012-9706-y).

71. Joehanes R, Nelson JC: QGene 4.0 an extensible Java QTL-analysis platform. Bioinformatics 2008, 24:2788-2789.

72. Voorrips RE: MapChart: Software for the graphical presentation of linkage maps and QTLs. J Hered 2002, 93(1):77-78.

doi:10.1186/s12870-014-0340-1

Cite this article as: Cabral et al: Identification of candidate genes, regions and markers for pre-harvest sprouting resistance in wheat (Triticum aestivum L.). BMC Plant Biology 2014 14:340.

\section{Submit your next manuscript to BioMed Central and take full advantage of:}

- Convenient online submission

- Thorough peer review

- No space constraints or color figure charges

- Immediate publication on acceptance

- Inclusion in PubMed, CAS, Scopus and Google Scholar

- Research which is freely available for redistribution 